\title{
European Society of Trauma and Emergency Surgery (ESTES) recommendations for trauma and emergency surgery preparation during times of COVID-19 infection
}

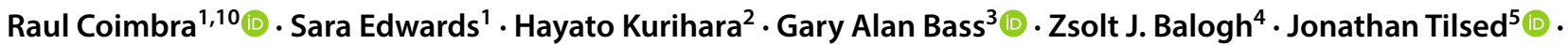 \\ Roberto Faccincani ${ }^{6}\left({ }^{10} \cdot\right.$ Michele Carlucci $^{6} \cdot{\text { Isidro Martínez } \text { Casas }^{7} \cdot \text { Christine Gaarder }^{8} \text {. Arnold Tabuenca }}^{1}$. \\ Bruno C. Coimbra ${ }^{1} \cdot$ Ingo Marzi $^{9}$
}

Received: 1 April 2020 / Accepted: 2 April 2020 / Published online: 17 April 2020

(c) Springer-Verlag GmbH Germany, part of Springer Nature 2020

\begin{abstract}
A series of recommendations regarding hospital perioperative preparation for the COVID-19 pandemic were compiled to inform surgeons worldwide on how to provide emergency surgery and trauma care during enduring times.The recommendations are divided into eight domains: (1) General recommendation for surgical services; (2) Emergency Surgery for critically ill COVID-19 positive or suspected patients -Preoperative planning and case selection; (3) Operating Room setup; (4) patient transport to the OR; (5) Surgical staff preparation; (6) Anesthesia considerations; (7) Surgical approach; and (8) Case Completion.The European Society of Emergency Surgery board endorsed these recommendations.
\end{abstract}

Keywords COVID-19 · Severe acute respiratory syndrome coronavirus 2 (SARS-CoV-2) · Trauma · Emergency surgery · Hospital preparation

Raul Coimbra

raulcoimbra62@yahoo.com

1 Comparative Effectiveness and Clinical Outcomes Research Center-CECORC, Riverside University Health System Medical Center, Moreno Valley, CA, USA

2 Emergency Surgery and Trauma Unit, Department of Surgery, Humanitas Research Hospital, Milan, Italy

3 Department of Surgery, Tallaght University Hospital, Dublin, Ireland

4 John Hunter Hospital and Hunter New England Health, University of Newcastle, Newcastle, NSW, Australia

5 Hull York Medical School, Heslington, England, UK

6 Emergency Department, IRCCS San Raffaele, Milan, Italy

7 Servicio de Cirugía General y Digestiva, Complejo Hospitalario de Jaén, Jaén, Spain

8 Department of Traumatology, Oslo University Hospital Ulleval, Oslo, Norway

9 Department of Trauma, Hand, and Reconstructive Surgery, University Hospital Frankfurt, Frankfurt, Germany

10 Loma Linda University School of Medicine, Loma Linda, USA

\section{Introduction}

Never before has the current generation of health care providers seen the dissemination of an infectious disease so devastating and widespread as the COVID-19 caused by the severe acute respiratory syndrome coronavirus 2 (SARSCoV-2). Initially affecting residents of Wuhan, China, in late December 2019, COVID-19 rapidly spread to every country on the globe. The consequences of this rapid spread, leading to patients with significant symptoms (particularly respiratory dysfunction or failure) to seek medical care in hospitals which, in normal circumstances, were already functioning at capacity. This surge of acute ill patients put a significant stress on the already overwhelmed health care system globally [1].

Despite the fact that no health care system was prepared for an event of this magnitude, hospitals and health care systems have started implementing measures to increase capacity to triage, test, cohort, and provide critical care services following current local guidelines, specific for each country or region $[2,3]$. As occurs in any other pandemic or mass casualty event, some patients with regular medical and surgical problems still require medical care. Many of those medical or surgical conditions may be treated at a later 
time when the pressure of COVID-19 eases; an example may include a myriad of general surgery problems treated electively [4]. To that end, many specialty societies have published their recommendations regarding postponement of what is considered "regular" or "elective" care [5, 6]. However, emergency general surgery conditions and trauma cases still require immediate assessment and timely resolution.

While disease specific guidelines have been published recently by many organizations, few recommendations have been made "by surgeons to surgeons" on the preparation of perioperative environments to support prompt care to emergency general surgery and trauma patients in times of COVID-19.

In response to a call from the Editor-in-Chief of the European Journal of Trauma and Emergency Surgery and the leadership of the European Society of Trauma and Emergency Surgery (ESTES), we develop this manuscript to inform Acute Care and Trauma Surgeons around the world about essential steps to prepare the surgical services of a hospital during these trying times.
The manuscript is a collection of measures implemented by front line surgeons in their health care facilities, regardless of being endorsed by governmental agencies or professional organizations. Some are real life lessons learned "on the spot", as many of us try to respond to the best of our ability to this pandemic. The content of the manuscript was approved by the ESTES Board and none of the authors reported any conflict of interest related to the manuscript.

We hope these measures and recommendations will help surgeons all over the world to lead in times of endurance and difficulty, and yet preserve and sustain adequate care for critically ill emergency general surgery and trauma patients.

\section{Recommendations for perioperative preparation}

See Tables 1, 2, 3, 4, 5, 6, 7, and 8 .

Table 1 General recommendations for surgical services

\footnotetext{
All elective surgeries should be postponed until further notice

It is possible that with the decrease in the number of elective surgical procedures and the need to increase Intensive Care Unit (ICU) bed capacity, surgical staff will be mobilized to provide non-surgical, COVID-19-related patient care. This shift of staff should not have a negative impact in our ability to provide timely care for trauma and emergency surgery patients

Cancer operations, patients with highly symptomatic benign disease, significant infections, and those whose delay would precipitate life-threatening outcomes or patient harm should be considered for operative intervention on a case-by-case basis by a multidisciplinary team including surgeons, anesthesiologists, and nursing leadership, depending on local availability of resources

Real-time reverse transcription polymerase chain reaction (RT-PCR) of viral nucleic acid is regarded as the reference standard in the diagnosis of SARS-CoV-2; however, delays in swab-to-result time may impact time-critical operative management of surgical conditions

The indications and principles of management for trauma and emergency general surgical operations are the same as in non-pandemic circumstances

Trauma and Emergency general surgery cases should proceed to the Operating Room in a timely manner with consideration to COVID-19 guidelines for symptomatic patients

Patients who do not have symptoms consistent with COVID-19, or have no radiologic findings, or have a negative RT-PCR test, should proceed to the OR with standard operating room precautions in place. Anesthesiologists may use N-93/FFP3 masks for intubations, if available

Patients with symptoms suggestive of COVID-19 infection who have a surgical condition requiring immediate attention and have not been tested prior to presentation to the ER, should undergo a chest X-ray and/or a chest Ultrasound and/or a Chest CT to look for bilateral interstitial pneumonitis (peripheral ground-glass consolidations) concerning for COVID-19 infection (Figs. 1, 2) [7-10]
} 
Table 2 Emergency surgery for critically ill COVID-19 positive or suspected patients—preoperative planning and case selection

Care should be taken to limit delay of interventions and to maintain quality of interventions

Cases should be reviewed by two surgeons (attendings/consultants), when available to confirm necessity and to weigh role for alternate interventions

If time permits, pre-operative risk stratification tools, such as p-POSSUM, POTTER and NELA, may guide recommendations regarding prognosis and hence appropriate goals of care [11]

Should delay to surgery not compromise care, cases should preferentially be performed at times of day when staffing and resources are optimal, and the test result or CT scan is available. This may preserve resources in cases that tested negative or a free of pulmonary infiltrates

Patients and families (by phone) should be engaged in robust goals of care conversations. (The recommendations below may not apply to certain countries)

Prior to operative intervention, document communication that delineates prognosis, goals of care, recommendations regarding interventions, and code status

All admitted patients, and particularly patients proceeding to OR should have consideration to code status and for those with anticipated poor prognosis, "Do Not Resuscitate" status should be considered preoperatively to limit the possibility of CPR in the OR

The use of vasopressors, in general, are used routinely as standard of care in anesthesia management, in general, and should be permitted following the standard of care

Trauma cases should be excluded from discussions regarding goals of care only if immediate surgery is required. Any advanced directives should be respected and reviewed

Table 3 Operating room (OR) setup

One or more dedicated operating rooms for COVID-19 positive cases must be identified

Efforts should be made to have the operating room conform to negative-pressure air flow. A high frequency air exchange ( $\geq 25$ cycles/hour) is preferred

Doors of the operating room should be closed at all times to maintain correct air flow

Anesthesia carts (with adequate medication stock), ventilators, cautery, laparoscopic towers, tables, and all essential equipment should be limited to use in the designated COVID-19 room

Terminal cleaning of all surfaces should be performed after each operation, following hospital guidelines

All surgical instruments used in the case should be covered for transport and returned for immediate sterilization following case completion

Individuals manipulating the used surgical instruments should handle with appropriate donning of PPE (gown, gloves, surgical mask)

All efforts should be made to have necessary equipment, including sutures and surgical instruments in the room for case start time, to limit entry and exit once case is underway

All instruments and supplies that have disposable alternatives should be employed

Table 4 Patient transport to the OR

All preoperative documentation is to be completed prior to transport of the patient into the operating room

Patients who do not require intubation prior to transport to the operating room must wear a surgical mask

Patients should be transported directly to the operating room, without stopping in the pre-operative holding area

Patients are to be transported in designated COVID elevator or designated pathways if available

Coordination between care teams is essential for safe transport

Peer to peer sign-out must occur by phone prior to transport (e.g., ER personnel to OR staff; surgeons to anesthesiologists)

Peer to peer handoff must occur upon arrival of the patient to the operating room

Staff transporting COVID-19 positive or suspected patients to the OR must wear N95/FFP3 masks, gown, and gloves to be removed when leaving the OR and placed in a designated receptacle

Hand washing should ALWAYS be performed after any doffing of PPE

Additional PPE should be made available for staff returning to wards

Staff transporting the patient will facilitate positioning patient on OR bed prior to exiting the room, to minimize the number of people in the room at one time 
Table 5 Surgical staff preparation

Surgical staff should be limited to essential personnel to avoid flux of multiple professionals into the room

PPE should be readily available at the door of the OR and donned prior to entry to the operating room

All personnel should wear an N95/FFP3 mask or, if available, PAPRs in the COVID-19 OR

Surgeons and scrub technicians/surgical assistants should wear an additional surgical mask over the N95/FFP3 mask to limit risk for splash contamination of the mask, which might necessitate mask exchange during the operation. Ideally, if available, a complete facial shield should be used over the two masks

All staff should be equipped with eye protection, masks, gown, gloves and shoe covers

Head coverage should include disposable cover

Personal reusable head coverage should be covered with a disposable cover

Personal fabric reusable head coverage is discouraged, unless strictly necessary in the absence of disposable head covers and should be washed and sanitized after each case

Surgeons, scrub technicians/surgical assistants, and nurses MUST double glove when scrubbed in the case

Standard sterile techniques should be employed in all cases

Operating room doors during operation should remain closed. If the operating room has two doors, one should be used exclusive for access to materials brought to the OR required during the case, which should be placed on a table and picked up by the staff inside the room to minimize person to person contact

A "runner" should remain available by phone to service the room and limit entrance and egress during the case

Table 6 Anesthesia considerations

Limiting the number of OR personnel in the room during intubation is advisable

Patients intubated in OR should have limited bagging, favoring Rapid Sequence Intubation (RSI)

Avoidance of awake intubation is recommended

Double gloves for intubation should be used routinely and the top layer removed following intubation to limit further contamination

Single attempt intubation should be sought

Endotracheal intubations should be performed by the most experienced individual in the OR setting. The practice of allowing junior residents and trainees to intubate patients in the COVID-19 OR is highly discouraged as multiple attempts to intubation increase the risk of unnecessary exposure of health care providers in the room

Bag Valve Mask (BVM) ventilation prior to intubation should be discouraged. If at all needed, use appropriate filters attached to the mask and secure the mask to the patient's face to avoid leaks and aerosolization

Video Laryngoscopy is preferred over Direct Laryngoscopy, when available

Fiberoptic intubation should be limited and avoided if possible

Disposable equipment should be used where applicable

The endotracheal tube cuff should be inflated before initiating mechanical ventilation

Closed suction systems should be used for airway aspiration and suctioning

Table 7 Surgical approach

Surgical approach should be dictated by best-practice accounting for reduced operative times and optimal surgical outcomes

The surgical procedure must be performed preferentially by an experienced surgeon. Avoiding using such cases for teaching purposes is highly advisable

In certain circumstances, alternatives to conventional surgical procedures may be considered depending on the clinical status of the patient

For cases performed laparoscopically, smoke evacuator attached to a HEPA filtration device must be used during the case and at the end of the case to facilitate release of pneumoperitoneum [12]

Smoke evacuators/filtration device should be used in all cases requiring electrocautery, laser, or ultrasonic scalpels, to limit exposure to aerosols [13-16]

The Neptune System, where available, may be utilized to permit a closed suction system, if available

All efforts should be made to limit the use of electrocautery, laser or ultrasonic scalpel to cases where non-aerosolizing techniques are available and confer acceptable outcomes 
Table 8 Case completion

Following completion of the operative case, patients should recover in the COVID-19 operating room until able to be transported directly to the appropriate unit outside of the operating room

Masks and disposable PPE should be removed, at case end, in accordance with standard doffing technique, witnessed in "buddy system" to facilitate removal, and disposed of in a lidded garbage can adjacent to the exit door of the operating room

Hand washing should occur immediately following doffing for all staff

Transporting OR staff are to maintain current mask but change gown and gloves for transport

Post-operative documentation should be performed outside of the Operating Room when possible

Peer-to-peer sign out will be conducted and the patient will return to the isolation ward or COVID-19 ICU

Following transport from the OR, mask should be disposed, and a new mask must be available for use

The surgical team should change OR scrubs immediately following the case

OR to ICU hand-off should proceed in accordance with the following:

Patients previously on medicine service should have postoperative care provided by the surgical teams in collaboration with primary team if necessary

Prioritize, as much as possible, admission to surgical service post-operatively

Continue isolation efforts during the post-operative period
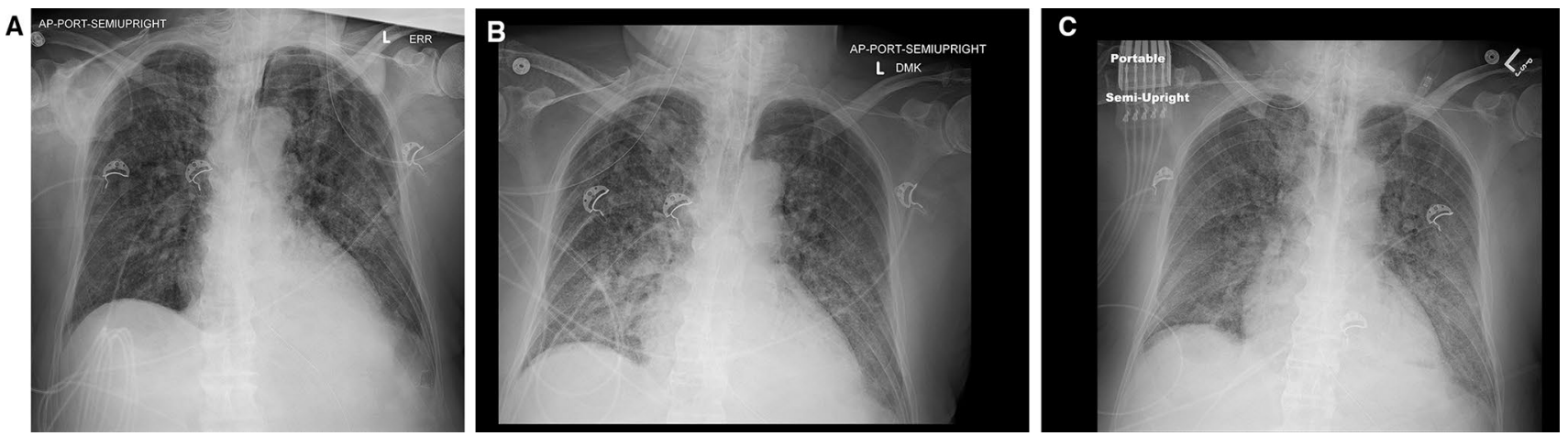

Fig. 1 Chest X-Ray of a symptomatic patient on hospital days 1 (a), 3 (b), and 5 (c). Note the rapid progression of the pulmonary infiltrates over time

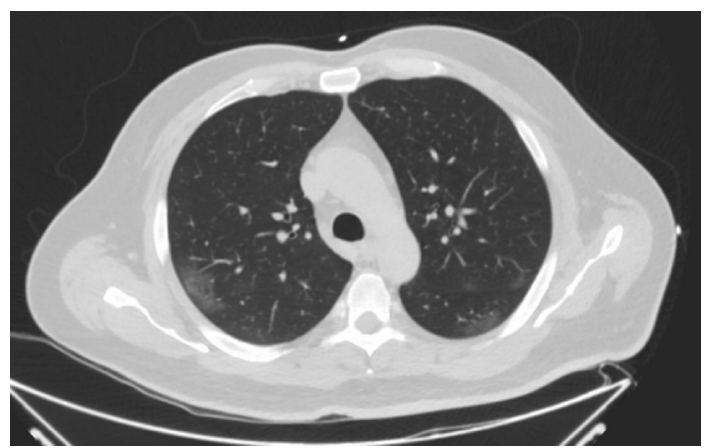

Fig. 2 Chest CT Image of an asymptomatic patient presenting with a strangulated hernia. CT findings immediately led to patient cohorting in COVID-19 unit post-operatively

\section{Final comments}

Surgeons will be asked to serve and lead during this pandemic. Maintaining our commitment to surgical patients is our obligation. We must maintain the same standards that we follow every day when treating patients in the trauma center or in the emergency department with surgical problems during these difficult times. No one believes it is easy to do, but it is our duty and our call. The best way to assure the public that we will be there for them, regardless of the circumstances, is to be prepared [17]. Learning from the experiences of many others and following the principles of personal protection, we will keep ourselves and our patients safe. 


\section{Compliance with ethical standards}

Conflict of interest The author declare that they have no competing interest.

\section{References}

1. Paules CI, Marston HD, Fauci AS. Coronavirus infections-more than just the common cold. JAMA. 2020;323(8):707-8.

2. Guidance for health system contingency planning during widespread transmission of SARS-CoV-2 with high impact on healthcare services. https://www.ecdc.europa.eu/sites/default/files/ documents/COVID-19-guidance-health-systems-contingenc y-planning.pdf. Accessed 30 Mar 2020.

3. Interim Guidance for Healthcare Facilities: Preparing for Community Transmission of COVID-19 in the United States. https:// www.cdc.gov/coronavirus/2019-ncov/healthcare-facilities/guida nce-hcf.html. Accessed 30 Mar 2020.

4. COVID-19: Recommendations for Management of Elective Surgical Procedures. March 13, 2020. https://www.facs.org/about-acs/ covid-19/information-for-surgeons/elective-surgery. Accessed 30 Mar 2020.

5. COVID-19 and Surgery: Resources for the Surgical Community. https://www.facs.org/covid-19. Accessed 30 Mar 2020.

6. SAGES COVID-19/Coronavirus announcements. https://www. sages.org/category/covid-19/. Accessed 30 Mar 2020.

7. Yoon SH, Lee KH, Kim JY, Lee YK, Ko H, Kim KH, Park CM, Kim YH. Chest radiographic and CT findings of the 2019 novel coronavirus disease (COVID-19): analysis of nine patients treated in Korea. Korean J Radiol. 2020;21:494-500.
8. Cheng Z, Lu Y, Cao Q, Qin L, Pan Z, Yan F, Yang W. Clinical features and chest CT manifestations of coronavirus disease 2019 (COVID-19) in a single-center study in Shanghai, China. AJR Am J Roentgenol. 2020;14:1-6. https://doi.org/10.2214/ajr.20.22959.

9. Ai T, Yang Z, Hou H, Zhan C, Chen C, Lv W, Tao Q, Sun Z, Xia L. Correlation of Chest CT and RT-PCR testing in coronavirus disease, 2019 (COVID-19) in China: a report of 1014 cases. Radiology. 2020. https://doi.org/10.1148/radiol.2020200642.

10. Buonsenso D, Pata D, Chiaretti A. COVID-19 outbreak: less stethoscope, more ultrasound. Lancet Respir Med. 2020. https:// doi.org/10.1016/S2213-2600(20)30120-X.

11. Bertsimas D, Dunn J, Velmahos G, Kaafarani H. Surgical risk is not linear. Ann Surg. 2018;268:574-83.

12. Resources for Smoke and Gas Evacuation During Open, Laparoscopic, and Endoscopic Procedures. https://www.sages.org/resou rces-smoke-gas-evacuation-during-open-laparoscopic-endoscopic -procedures/. Accessed 30 Mar 2020.

13. Alp E, Bijl D, Bleichrodt RP, Hansson B, Voss A. Surgical smoke and infection control. J Hosp Infect. 2006;62(1):1-5.

14. Bigony L. Risks associated with exposure to surgical smoke plume: a review of the literature. AORN J. 2007;86(6):1013-20.

15. Karsai S, Däschlein G. "Smoking guns": hazards generated by laser and electrocautery smoke. J Dtsch Dermatol Ges. 2012;10(9):633-6.

16. Elmashae Y, Koehler RH, Yermakov M, Reponen T, Grinshpun SA. Surgical smoke simulation study: physical characterization and respiratory protection. Aerosol Sci Technol. 2018;52(1):38-45.

17. Kurihara H, Bisagni P, Faccincani R, Zago M. Covid-19 outbreak in Northern Italy: viewpoint of the Milan Area Surgical Community. J Trauma Acute Care Surg. 2020 (in press). 